\title{
THE FUTURE SCIENCE
}

The current thrust of scientific discourse in biological science is centered on exploration of the intrinsic factors directly involved in facilitating the life, and determining other ancillary factors maintaining it in proper order for conglomeration into the concrete life system. One of the trademark breakthroughs has been the Venterian artificial life, in which whole life was created artificially mediated by genetic reconstitution in scaffold of cellular system. Similarly, rapid interest and flux of cutting edge research is centered to understanding and thus devising mechanism to control the metabolic and physiological profile to prevent various diseases such as Alzheimer's disease, Parkinson disease, melanomas, sarcomas etc. In this effort whole consortium of scientific community, academicians and researchers are putting their hands together for creation of panoramic globe, where there is cure for every kind of diseases or disorders.

One of the most important aspects associated with the fast moving technological intervention has been co-ordination, co-operation and integration of scientific communities, for which the current communication technology is responsible. Due to amalgamation of ideas and knowledge of scientists across the cross-country boundaries, the scientific pulse is rapidly increasing. But still in this phase of transition of world, it can be felt that some patch on the globe is still undermined and to some extent this part is unaware of the technological charisma. But, this part too has some crucial contribution when conclusion has to be summed up taking into consideration of the whole globe as a single system.

The crux of technological impetus has been the utilization of natural resources, so the developed community is moving forward for the world with flux of artificial stuffs. The current research in top tier economic colossus is pivoted on automation with robotics, complementation and supplementation of requisites with artificial stuffs, and designing of efficient strategies to curtail the natural requirements. But, regarding the sustainability it is still questionable in terms of rationality. In contrast, on the backdoor we can realize that undermined patch of world is still furbished with enormous natural resource in terms of flora and fauna. It is unquestionable that we can go for sustainable use of the natural resources rather than purging all our effort in artifice creation. It is more rational to devise the strategic approaches to maintain the sustainable utilization of the natural resources available. For that the idea from the developed community should be mingled with the natural resources available in hotspots patches of globe, to create a utopian era. Hence, the future science should be BE NATURAL WITH SUSTAINABILITY!

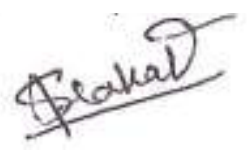

Dipesh Dhakal

Executive editor

Nepal Journal of Biotechnology 